\section{Efectividad del ejercicio físico en la fatiga de pacientes con cáncer durante el tratamiento activo: revisión sistemática y metaanálisis}

\author{
Effectiveness of physical exercise on fatigue \\ in cancer patients during active treatment: \\ a systematic review and meta-analysis
}

\author{
Eficácia do exercício físico na fadiga dos pacientes \\ com câncer durante o tratamento ativo: revisão \\ sistemática e meta-análise
}

\author{
${ }^{1}$ Facultad de Cultura \\ Física, Deporte y Recreación, \\ Universidad Santo Tomás, \\ Bogotá, Colombia. \\ 2 Escuela de Medicina \\ y Ciencias de la Salud, \\ Universidad del Rosario, \\ Bogotá, Colombia. \\ 3 Facultad de Enfermería, \\ Universidad de Granada, \\ Granada, España. \\ Correspondencia \\ J. F. Meneses-Echávez \\ Grupo GICAEDS, Facultad \\ de Cultura Física, Deporte \\ y Recreación, Universidad \\ Santo Tomás. \\ Carrera 9 \# 51-11, Bogotá, \\ D.C., Colombia. \\ menesesjose77@gmail.com
}

\begin{abstract}
This study aimed to determine the effectiveness of physical exercise in decreasing fatigue in cancer patients during active treatment. The PubMed Central, EMBASE, and OVID databases were consulted up to April 2014 to identify randomized clinical trials that evaluated the effect of exercise on fatigue in cancer patients undergoing active treatment. Eleven studies ( $n=1,407)$ were included. Chemotherapy was the most common form of treatment $(n=1,028)$. The studies showed a low risk of bias and high methodological quality. Effect estimates showed that physical exercise significantly improved fatigue (SMD =-3.0; 95\%CI: -5.21;-0.80), $p<0.0001$. Similar effects were found for resistance training (SMD = -4.5; 95\%CI: $-7.24 ;-1.82$ ), $p=0.001$. Significant improvements were found in breast and prostate cancer patients $(p<0.05)$. Exercise is a safe and effective intervention in the management fatigue in cancer patients undergoing active treatment.
\end{abstract}

Exercise; Fatigue; Neoplasms; Drug Therapy
Jose Francisco Meneses-Echávez 1,2 Emilio González-Jiménez 3 Jorge Enrique Correa-Bautista ${ }^{2}$ Jacqueline Schmidt-Río Valle 3 Robinson Ramírez-Vélez ${ }^{1,2}$

\section{Resumen}

El objetivo del estudio fue determinar la efectividad del ejercicio físico en la fatiga de pacientes con cáncer durante el tratamiento activo. Las bases de datos de PubMed Central, EMBASE y OVID fueron consultadas hasta abril de 2014 para identificar ensayos clínicos aleatorizados, que evaluaran el efecto del ejercicio en la fatiga de pacientes con cáncer sometidos a tratamiento activo. Once estudios ( $n=1.407)$ fueron incluidos. La quimioterapia fue el tratamiento más común ( $n=1.028)$. Los estudios tuvieron bajo riesgo de sesgo y alta calidad metodológica. Las estimaciones de efecto mostraron que el ejercicio físico mejoró significativamente la fatiga (SMD = -3,0; IC95\%: -5,21; -0,80), $p<0,0001$. Se encontraron efectos similares para el entrenamiento de resistencia (SMD = -4,5; IC95\%: $-7,24 ;-1,82)$, $p=0,001$. Se encontraron mejoras significativas en pacientes con cáncer de mama y de próstata ( $p<0,05)$. El ejercicio es una intervención segura y eficaz en el control de la fatiga en pacientes sometidos a tratamiento activo.

Ejercicio; Fatiga; Neoplasias; Quimioterapia 


\section{Introducción}

El cáncer es un problema de salud pública en el mundo. Se estima que cada año se presentan cerca de 11 millones de casos nuevos a nivel mundial, de los cuales el $80 \%$ se dan en países en vía de desarrollo ${ }^{1}$. Según datos del Instituto Nacional de Cancerología (INC), los tumores son la tercera causa de muerte en Colombia; tendencia que ha aumentado en las últimas décadas desde un $6 \%$ a un $15 \% 2$. El actual modelo para el control del cáncer en Colombia, basado en las directrices de la Organización Mundial de la Salud (OMS) y adoptado en la mayoría de países del mundo, tiene por objetivos el control del riesgo, la detección temprana, el tratamiento y rehabilitación, y el cuidado paliativo ${ }^{3}$. Dentro del cual, las intervenciones no farmacológicas tales como el ejercicio físico cuentan con un amplio cuerpo de evidencia científica 4,5 .

La fatiga relacionada al cáncer es el efecto colateral más común del tratamiento del cáncer, con prevalencias que van del $60 \%$ al $96 \%$ en pacientes sometidos a tratamiento activo 6 . La fatiga puede persistir durante meses e incluso años después del tratamiento exitoso ${ }^{6,7}$. La Red Nacional de Cáncer de los Estados Unidos (en inglés, NCCN) 7 define la fatiga como "una sensación subjetiva y persistente de cansancio fisico, emocional y/o cognitivo, relacionada con el tratamiento del cáncer, que no es proporcional a la actividad reciente y que interfiere con la funcionalidad diaria". La fatiga impacta en las actividades diarias, las relaciones sociales y la calidad de vida del paciente con cáncer 8 e incluso ha sido postulada como un predictor de supervivencia en estos pacientes 9 .

El ejercicio físico es considerado una intervención no farmacológica eficaz en la promoción del bienestar físico, mental y funcional de pacientes con cáncer 10,11,12. Cramp \& Byron 13 en una revisión sistemática encontraron que el ejercicio físico produjo mejoras significativas en la fatiga de pacientes con cáncer durante y después del tratamiento activo; aunque los autores no realizaron estimaciones del efecto de las modalidades de ejercicio estudiadas. Recientemente, Lin et al. 14 demostraron que 12 semanas de ejercicio físico mejoraron los niveles de fatiga, la calidad de vida, la fuerza muscular y el fitness cardiorrespiratorio en pacientes con cáncer colorrectal durante la quimioterapia. Estos hallazgos han sido apoyados por otras investigaciones 15,16. Sin embargo, la evidencia se muestra contradictoria acerca de los efectos y de la seguridad de las intervenciones con ejercicio físico durante el tratamiento activo de pacientes con cáncer. Por lo tanto, el objetivo de esta revisión sistemática y meta-análisis es determinar la efectividad del ejercicio físico, incluyendo el entrenamiento de resistencia, en la fatiga de pacientes con cáncer durante el tratamiento activo.

\section{Métodos}

La presente revisión sistemática fue desarrollada según las directrices de la declaración PRISMA 17 y las recomendaciones de la Colaboración Cochrane para la conducción de revisiones sistemáticas de estudios experimentales 18 .

\section{Estrategia de búsqueda}

Se consultaron las bases de datos de PubMed Central, EMBASE y OVID durante los meses de febrero y abril de 2014 por parte de dos autores independientes y cegados (J.F.M.-E. y R.R.-V.). La estrategia de búsqueda, combinando el uso de operadores booleanos AND y OR, fue la siguiente: (randomized controlled trial) OR controlled clinical trial) OR randomized) OR trial) OR "clinical trials as topic") AND cancer) OR neoplasm*) OR tumour*) OR tumor*) OR carcino*) OR leukaemi*) OR leukemi*) AND chemotherapy) OR radiotherapy) OR radiation) AND) OR exercise) OR physical activity) OR aerobic) OR resistance) OR strength) AND fatigue). Otros dos autores independientes (J.E.C.-B., J.S.R.-V.) revisaron las listas de referencias de los estudios identificados, las memorias de las reuniones anuales de la Sociedad Americana del Cáncer (en inglés ASCO) y la Sociedad Europea de Oncología Médica (en inglés ESMO), y los números de algunas revistas científicas tales como Cancer, Journal of Clinical Oncology, Breast Cancer Research, Journal of Oncology Practice y The Lancet Oncology. Las búsquedas se delimitaron a estudios publicados desde 2000 a 2014, ya que investigaciones previas han demostrado algunas limitaciones metodológicas en los estudios publicados antes de 2000, especialmente en lo relacionado a la aleatorización y la medición de desenlaces. Para controlar un posible sesgo idiomático, los autores no aplicaron restricciones idiomáticas a las búsquedas.

\section{Criterios de selección}

Los criterios de selección fueron definidos con base en el acrónimo PICO 18. Los criterios de selección fueron aplicados por dos autores independientes (J.F.M.-E., J.E.C.-B.), de manera cegada. Un tercer autor medió, en caso de ser necesario, para lograr consenso (R.R.-V.). 


\section{- Participantes}

Pacientes adultos (edad > 18 años) con diagnóstico cáncer, sin delimitación a un estadio de progresión específico. No se realizaron filtros según régimen de tratamiento (quimioterapia, radioterapia, suplemento hormonal u otro), sexo, raza ni etnia. Un autor (E.G.-J.) verificó el cumplimiento de las recomendaciones éticas para la investigación en humanos en cada uno de los estudios incluidos.

\section{- Intervención}

Se consideraron intervenciones con ejercicio físico que siguieran la definición propuesta por Wolin et al. 19: cualquier movimiento corporal estructurado que aumenta el gasto energético, llevado a cabo de manera sistemática en términos de frecuencia, intensidad y duración, y que es diseñado para mantener y mejorar la salud. En este sentido, se incluyeron intervenciones de ejercicio físico supervisadas por profesionales de la salud que incluyeran ejercicio aeróbico, ejercicios de fortalecimiento muscular, también conocido como entrenamiento de resistencia, y los programas de estiramiento musculoesquelético. Estas intervenciones han sido propuestas por el Colegio Americano de Medicina Deportiva (en inglés ACSM) 20. Por otra parte, se excluyeron las intervenciones que incluyeran yoga, el tai-chi y otras de terapia manual, ya que al no producir efectos significativos en el aumento del gasto energético, no se ajustan a los criterios de selección definidos a priori. Sólo se consideraron para su inclusión aquellos ensayos clínicos aleatorizados que fueran realizados durante el tratamiento activo.

\section{- Medidas de resultado}

La medida de resultado primaria fue la fatiga relacionada al cáncer, ajustada a la definición de la NCCN 7 y evaluada mediante instrumentos validados tales como la Escala de Fatiga de la Evaluación Funcional de la Terapia del Cáncer (en inglés FACT-Fatigue Scale); el Cuestionario para la Evaluación de la Calidad de Vida en Cáncer de la Organización Europea para la investigación y el tratamiento del cáncer (en inglés EORTC QLQ-C30), la Escala de Fatiga de Piper (en inglés PFS); la Escala de Schwartz para Fatiga (en inglés SCFS) y el Instrumento Multidimensional de Fatiga (en inglés MFI). Como medidas de resultado secundarias se incluyeron los reportes de bienestar físico y bienestar funcional, ya que estas se relacionan de manera directa con los síntomas de fatiga, controlando las estimaciones de efecto a obtener.
Evaluación del riesgo de sesgo en los estudios incluidos

El riesgo de sesgo fue evaluado usando la escala de PEDro 21 (en inglés Physiotherapy Evidence Database). La escala de PEDro consta de 10 ítems que evalúan la validez interna (criterios 2-9) y la información estadística de los ensayos clínicos (criterios 10 y 11). Cada ítem se suma contando 1 como presente o ausente. Moseley et al. 22 reportan que los estudios con puntuaciones iguales o superiores a $5 / 10$ pueden ser considerados como estudios de alta calidad metodológica y bajo riesgo de sesgo. Este criterio se incorporó para efectos del presente estudio. Tres autores (J.F.M.-E., J.E.C.-B. y E.G.-J.) realizaron de manera independiente y cegada esta etapa de la revisión.

\section{Extracción de datos y plan de análisis}

Dos autores (J.E.C.-B. y E.G.-J.) realizaron los procesos de extracción de datos de manera independiente. Se extrajeron datos sobre las siguientes características: información de los autores, año de publicación, muestra y asignación, características de las intervenciones (intensidad, frecuencia y duración), modalidad(es) de ejercicio físico implementadas, medidas de resultado y conclusiones. Los datos fueron registrados en un formulario electrónico estándar y verificados por un tercer autor (R.R.-V.).

Para el análisis combinado se calcularon diferencias de media estandarizadas (SMD) con el respectivo intervalo de confianza al 95\% (IC95\%) de precisión y un valor de significancia de $\mathrm{p}<$ 0,05 . El estadístico I2 fue utilizado para evaluar la heterogeneidad estadística, tomando los siguientes puntos de corte: $0-40 \%$ heterogeneidad leve, $40 \%-70 \%$ heterogeneidad moderada y $70 \%-100 \%$ alta heterogeneidad 18,23. Se empleó un modelo de efectos fijos en ausencia de heterogeneidad ( $\mathrm{I}^{2}<50 \%$ ); de lo contrario se seleccionó un modelo de efectos aleatorios. De igual forma, se calcularon estimaciones de efecto para el entrenamiento de resistencia y para los programas de estiramiento. Se realizó un análisis de subgrupos, según la localización del cáncer, y según la modalidad de ejercicio investigada, para lo cual se obtuvieron estimaciones de efecto con el modelo de análisis descrito anteriormente. El sesgo de publicación fue evaluado mediante análisis visual del gráfico de embudo (en inglés funnel plot). Los análisis fueron realizados por un autor independiente (J.F.M.-E.). Para el cálculo de las estimaciones de efecto se empleó el software Stata, version 12.0 (Stata Corp., College Station, EEUU). 


\section{Resultados}

\section{Características de los estudios incluidos}

Se localizaron 8.557 títulos, de los cuales tras la aplicación de los criterios de selección definidos, sólo 11 estudios $(n=1.407)$ fueron incluidos en el presente estudio $24,25,26,27,28,29,30,31,32,33,34$. La Figura 1 presenta el diagrama de flujo con los procesos de búsqueda y selección de los estudios incluidos. Los estudios presentaron un bajo riesgo de sesgo y una alta calidad metodológica [puntuación PEDro = 6,5 (DE \pm 1 )]. La evaluación del sesgo se presenta en la Tabla 1.

Solo tres estudios reportaron el seguimiento realizado en sus metodologías 26,30,31. Courneya et al. 26 encontraron que las diferencias en la fatiga, el funcionamiento físico y la calidad de vida no fueron significativas a los 6 meses de seguimiento post-intervención $(\mathrm{p}=0,054)$. Milne et al. 30 obtuvieron datos de seguimiento en el $97 \%$ de los participantes con una adherencia del $61,3 \%$, registrando mejoras estadísticamente significativas en la fatiga, la calidad de vida, la ansiedad y el fitness físico $(\mathrm{p}<0,05)$. Por último, Mutrie et al. 31 encontraron que los efectos del ejercicio físico en la fatiga del grupo experimental se mantuvieron durante los 6 meses de seguimiento $(p<0,05)$ Las características de los estudios incluidos se presentan en la Tabla 2.

\section{Características de los participantes}

Los participantes $(\mathrm{n}=1.407)$ tuvieron una edad de 55,5 $\pm 7,2$ años. Un total de 1.353 participantes fueron mujeres (86\%). El tratamiento más común fue la quimioterapia $(n=1.028)$. El tiempo desde el diagnóstico fue 8,2 $\pm 10,7$ meses, aunque éste no fue reportado en todos los estudios. Por otra parte, el cáncer de mama fue el tipo de dolencia más estudiado $(n=6) 25,26,30,31,32,33$, seguido del cáncer de próstata $(n=2)$ 29,35 y linfoma 27; mientras que dos estudios incluyeron pacientes con diferentes tipos de cáncer 24,28.

Figura 1

Diagrama de flujo PRISMA para la búsqueda y selección de los estudios primarios.

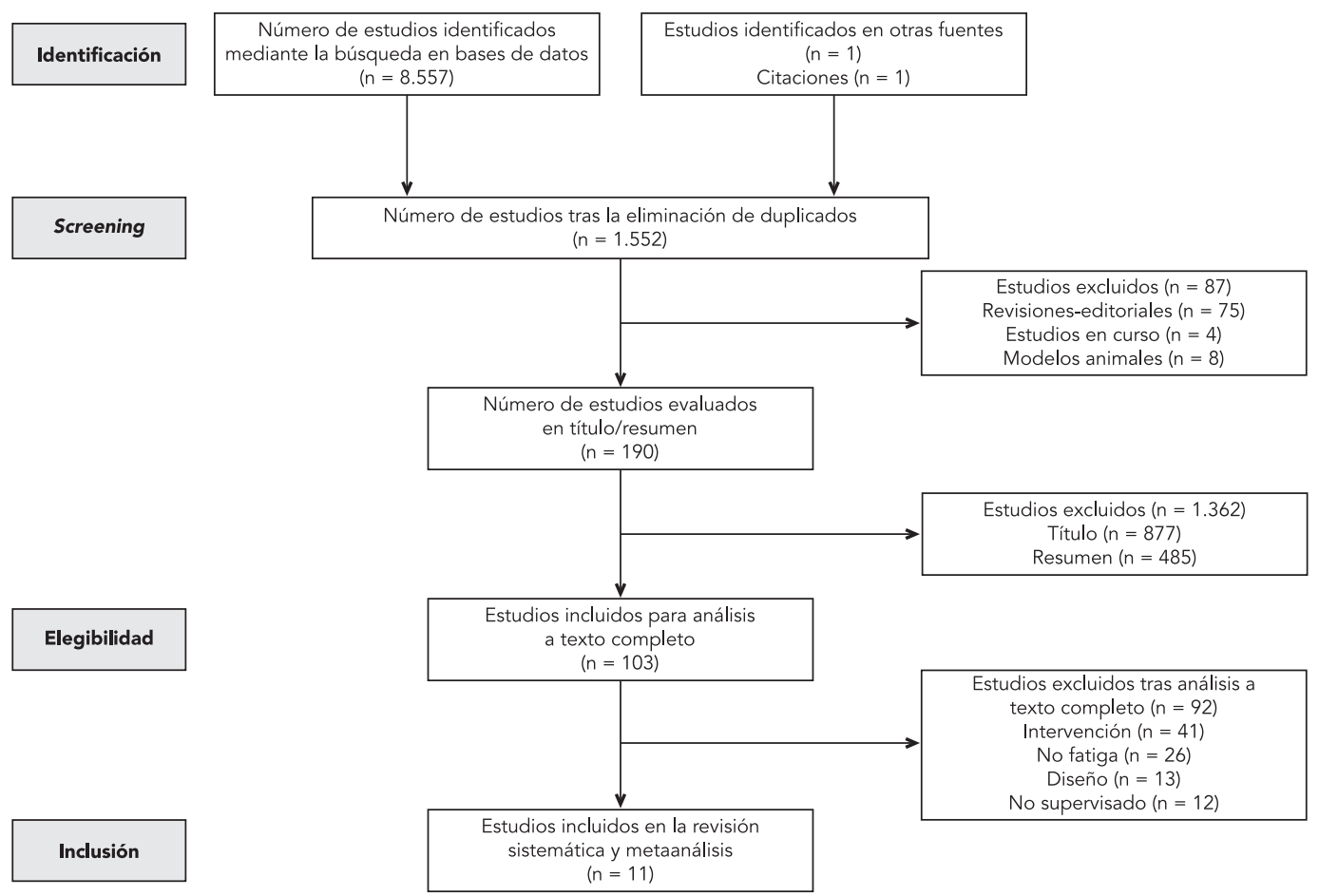


Tabla 1

Evaluación del riesgo de sesgo usando la escala de PEDro $(n=11)$.

\begin{tabular}{|c|c|c|c|c|c|c|c|c|c|c|c|}
\hline Referencia & P1 & P2 & P3 & P4 & P5 & P6 & P7 & P8 & P9 & P10 & Total \\
\hline Adamsen et al. 24 & + & + & + & - & - & - & + & + & + & + & 7 \\
\hline Campbell et al. 25 & + & - & + & - & - & - & + & - & + & + & 5 \\
\hline Courneya et al. 26 & + & - & + & - & - & + & + & + & + & + & 7 \\
\hline Courneya et al. 27 & + & + & + & - & - & - & + & + & + & + & 7 \\
\hline Dimeo et al. 28 & + & + & + & - & - & - & + & - & + & + & 6 \\
\hline Galvão et al. 29 & + & + & + & - & - & - & + & + & + & + & 7 \\
\hline Milne et al. 30 & + & + & + & - & - & - & + & + & + & + & 7 \\
\hline Mutrie et al. 31 & + & + & + & - & - & + & + & + & + & + & 8 \\
\hline Saarto et al. 32 & + & - & + & - & - & - & + & - & + & + & 5 \\
\hline Segal et al. 33 & + & - & + & - & - & - & - & + & + & + & 5 \\
\hline Segal et al. 34 & + & + & + & - & - & - & + & + & + & + & 7 \\
\hline
\end{tabular}

PEDro (Physiotherapy Evidence Database): + Sí; - No.

P1: Asignación aleatoria; P2: Ocultamiento de la asignación; P3: Grupos similares en línea de base; P4: Cegamiento de los participantes; P5: Cegamiento de los terapeutas; P6: Cegamiento del evaluador; P7: Abandonos < 15\%; P8: Análisis por intención a tratar; P9: Diferencias reportadas entre grupos; P10: Punto estimado y variabilidad reportada.

Tabla 2

Características de los estudios incluidos en la revisión sistemática y metaanálisis.

\begin{tabular}{|c|c|c|c|c|c|}
\hline Estudio & Diseño & $\begin{array}{l}\text { Tipo de } \\
\text { cáncer }\end{array}$ & Participantes (n) * & Intervención ** & Medición de resultados \\
\hline $\begin{array}{l}\text { Adamsen } \\
\text { et al. } 24\end{array}$ & $\begin{array}{l}\text { Ensayo clínico } \\
\text { aleatorizado }\end{array}$ & Mixto & $\begin{array}{l}\text { Características del tratamiento } \\
\text { de cáncer: } \\
\text { Quimioterapia }=235 \\
\text { Femenino }=171 \\
\text { Masculino }=64 \\
\text { Grupo experimental }=118 \\
\text { Edad (años) }=47,2(10,7) \\
\text { Grupo control }=117 \\
\text { Edad (años) }=47,2(10,6)\end{array}$ & $\begin{array}{l}\text { Grupo experimental = ejercicio } \\
\text { aeróbico, entrenamiento de } \\
\text { resistencia y estiramiento } \\
\text { Tiempo }=6 \text { semanas } \\
\text { Duración }=120 \text { minutos/sesión } \\
\text { Frecuencia }=5 \text { sesiones } / \text { semana } \\
\text { Intensidad }=85 \%-95 \% \\
\text { Grupo control = cuidado } \\
\text { convencional }\end{array}$ & $\begin{array}{c}\text { EORTC QLQ-C30, } \\
\text { MOS SF-36, Tiempo de } \\
\text { ocio, Cuestionario de } \\
\text { actividad física, Fuerza } \\
\text { muscular (1RM) }\end{array}$ \\
\hline $\begin{array}{l}\text { Campbell } \\
\text { et al. } 25\end{array}$ & $\begin{array}{c}\text { Ensayo clínico } \\
\text { aleatorizado }\end{array}$ & $\begin{array}{c}\text { Cáncer de } \\
\text { mama estadio } \\
\text { temprano (I-II) }\end{array}$ & $\begin{array}{l}\text { Características del tratamiento } \\
\text { de cáncer: } \\
\text { Quimioterapia, radioterapia y } \\
\text { combinación }=22 \\
\text { Femenino }=22 \\
\text { Grupo experimental }=12 \\
\text { Edad (años) }=48(10) \\
\text { Grupo control }=10 \\
\text { Edad (años) }=47(5)\end{array}$ & $\begin{array}{c}\text { Grupo experimental = ejercicio } \\
\text { aeróbico y entrenamiento de } \\
\text { resistencia } \\
\text { Tiempo }=12 \text { semanas } \\
\text { Duración }=20 \text { minutos } / \text { sesión } \\
\text { Frecuencia }=2 \text { sesiones } / \text { semana } \\
\text { Intensidad }=60 \%-75 \% \\
\text { Grupo control = cuidado } \\
\text { convencional }\end{array}$ & $\begin{array}{c}\text { FACT-G, FACT-B, SWLS, } \\
\text { PFS, SPAQ, Test de } \\
\text { caminata } 12 \text { minutos }\end{array}$ \\
\hline $\begin{array}{l}\text { Courneya } \\
\text { et al. } 26\end{array}$ & $\begin{array}{l}\text { Ensayo clínico } \\
\text { aleatorizado }\end{array}$ & $\begin{array}{c}\text { Cáncer de } \\
\text { mama estadio } \\
\text { temprano (I-II) }\end{array}$ & $\begin{array}{c}\text { Características del tratamiento } \\
\text { de cáncer } \\
\text { Quimioterapia y } \\
\text { radioterapia }=52 \\
\text { Femenino }=52 \\
\text { Grupo experimental }=24 \\
\text { Edad (años) }=59(5) \\
\text { Grupo control }=28 \\
\text { Edad (años) }=58(6)\end{array}$ & $\begin{array}{c}\text { Grupo experimental = ejercicio } \\
\text { aeróbico } \\
\text { Tiempo }=15 \text { semanas } \\
\text { Duración }=35 \text { minutos } / \text { sesión } \\
\text { Frecuencia }=3 \text { sesiones } / \text { semana } \\
\text { Intensidad }=70 \%-75 \% \\
\text { Grupo control }=\text { cuidado } \\
\text { convencional }\end{array}$ & $\begin{array}{c}\text { FACT- G, FACT-B, } \\
\text { FACT-F }\end{array}$ \\
\hline
\end{tabular}

(continua) 
Tabla 2 (continuación)

\begin{tabular}{|c|c|c|c|c|c|}
\hline Estudio & Diseño & $\begin{array}{l}\text { Tipo de } \\
\text { cáncer }\end{array}$ & Participantes $(n)$ * & Intervención ** & Medición de resultados \\
\hline $\begin{array}{l}\text { Courneya et } \\
\text { al. } 27\end{array}$ & $\begin{array}{l}\text { Ensayo clínico } \\
\text { aleatorizado }\end{array}$ & $\begin{array}{l}\text { Linfoma } \\
\text { etapas I-IV, } \\
\text { Linfoma de } \\
\text { Hodgkin } \\
(18 \%) \text { y } \\
\text { Linfoma } \\
\text { No-Hodgkin } \\
(82 \%)\end{array}$ & $\begin{array}{l}\text { Características del tratamiento } \\
\text { de cáncer } \\
\text { Quimioterapia }=122 \\
\text { Femenino }=50 \\
\text { Masculino }=72 \\
\text { Grupo experimental }=60 \\
\text { Edad (años) }=52,8(18-77) \\
\text { Grupo control }=62 \\
\text { Edad (años) }=53,5(18-80)\end{array}$ & $\begin{array}{c}\text { Grupo experimental }= \\
\text { ejercicio aeróbico } \\
\text { Tiempo }=12 \text { semanas } \\
\text { Duración }=45 \text { minutos } / \text { sesión } \\
\text { Frecuencia }=3 \text { sesiones } / \text { semana } \\
\text { Intensidad }=60 \%-75 \% \\
\text { Grupo control }=\text { cuidado } \\
\text { convencional }\end{array}$ & FACT-G, FACT-F \\
\hline $\begin{array}{l}\text { Dimeo et } \\
\text { al. } 28\end{array}$ & $\begin{array}{l}\text { Ensayo clínico } \\
\text { aleatorizado }\end{array}$ & $\begin{array}{l}\text { Tipo mixto } \\
\text { de cáncer } \\
\text { estadios } \\
\text { tumorales I-IV }\end{array}$ & $\begin{array}{c}\text { Características del tratamiento } \\
\text { de cáncer } \\
\text { Quimioterapia y } \\
\text { radioterapia }=69 \\
\text { Femenino }=20 \\
\text { Masculino }=49 \\
\text { Grupo experimental }=34 \\
\text { Edad (años) }=55,1(10) \\
\text { Grupo control }=35 \\
\text { Edad (años) }=60(9,5)\end{array}$ & $\begin{array}{c}\text { Grupo experimental }= \\
\text { ejercicio aeróbico } \\
\text { Tiempo }=3 \text { semanas } \\
\text { Duración }=30 \text { minutos } / \text { sesión } \\
\text { Frecuencia }=5 \text { sesiones/semana } \\
\text { Intensidad }=\text { sobre } 80 \% \\
\text { Grupo control }=\text { entrenamiento de } \\
\text { relajación progresiva ( } 45 \text { minutos/ } \\
\text { tres veces por semana) }\end{array}$ & EORTC QLQ-C3O \\
\hline $\begin{array}{l}\text { Galvão et } \\
\text { al. } 29\end{array}$ & $\begin{array}{l}\text { Ensayo clínico } \\
\text { aleatorizado }\end{array}$ & $\begin{array}{c}\text { Localizados } \\
\text { en próstata } \\
(93,1 \%), \\
\text { Metástasis } \\
\text { ganglionares } \\
(6,9 \%)\end{array}$ & $\begin{array}{c}\text { Características del tratamiento } \\
\text { de cáncer } \\
\text { Quimioterapia y } \\
\text { radioterapia }=57 \\
\text { Masculino }=57 \\
\text { Grupo experimental }=29 \\
\text { Edad (años) }=53,5(8,7) \\
\text { Grupo control }=28 \\
\text { Edad (años) }=52,1(11,8)\end{array}$ & $\begin{array}{l}\text { Grupo experimental = ejercicio } \\
\text { aeróbico, entrenamiento de } \\
\text { resistencia y estiramiento } \\
\text { Tiempo }=12 \text { semanas } \\
\text { Duración }=20 \text { minutos/sesión } \\
\text { Frecuencia }=2 \text { sesiones } / \text { semana } \\
\text { Intensidad }=65 \%-80 \% . \\
\text { Grupo control = cuidado } \\
\text { convencional }\end{array}$ & $\begin{array}{c}\text { EORTC QLQ-C30, MOS } \\
\text { SF-36, DEXA, 1RM }\end{array}$ \\
\hline Milne et al. 30 & $\begin{array}{l}\text { Ensayo clínico } \\
\text { aleatorizado }\end{array}$ & $\begin{array}{c}\text { Cáncer de } \\
\text { mama estadio } \\
\text { temprano (I-II) }\end{array}$ & $\begin{array}{c}\text { Características del tratamiento } \\
\text { de cáncer } \\
\text { Quimioterapia y } \\
\text { radioterapia }=58 \\
\text { Femenino }=58 \\
\text { Grupo experimental }=29 \\
\text { Edad (años) }=55,2(8,4) \\
\text { Grupo control }=29 \\
\text { Edad (años) }=55,1(8,0)\end{array}$ & $\begin{array}{c}\text { Grupo experimental = ejercicio } \\
\text { aeróbico, entrenamiento de } \\
\text { resistencia y estiramiento } \\
\text { Tiempo = } 12 \text { semanas } \\
\text { Duración }=30 \text { minutos/sesión } \\
\text { Frecuencia }=3 \text { sesiones/semana } \\
\text { Intensidad = sobre } 75 \% . \\
\text { Grupo control = grupo de ejercicio } \\
\text { retardado (GER) completaron el } \\
\text { programa de ejercicio de } 13 \text { a } 24 \\
\text { semanas }\end{array}$ & $\begin{array}{c}\text { FACT-B, SCFS, rPARQ, } \\
\text { Índice de potencia } \\
\text { aeróbica }\end{array}$ \\
\hline Mutrie et al. 31 & $\begin{array}{l}\text { Ensayo clínico } \\
\text { aleatorizado }\end{array}$ & $\begin{array}{c}\text { Cáncer de } \\
\text { mama estadio } \\
\text { temprano (I-II) }\end{array}$ & $\begin{array}{l}\text { Características del tratamiento } \\
\text { de cáncer } \\
\text { Quimioterapia, radioterapia y } \\
\text { combinación }=174 \\
\text { Femenino }=174 \\
\text { Grupo experimental }=82 \\
\text { Edad (años) }=51,3(10,3) \\
\text { Grupo control }=92 \\
\text { Edad (años) }=51,8(8,7)\end{array}$ & $\begin{array}{c}\text { Grupo experimental = ejercicio } \\
\text { aeróbico y entrenamiento de } \\
\text { resistencia } \\
\text { Tiempo }=12 \text { semanas } \\
\text { Duración }=45 \text { minutos } / \text { sesión } \\
\text { Frecuencia }=2 \text { sesiones } / \text { semana } \\
\text { Intensidad }=50 \%-75 \% \\
\text { Grupo control = cuidado } \\
\text { convencional }\end{array}$ & $\begin{array}{c}\text { FACT-G, FACT-B, } \\
\text { FACT-F, BDI, PANAS, } \\
\text { SPAQ Tiempo de ocio, } \\
\text { IMC, Test de caminata } \\
\text { de } 12 \text { minutos }\end{array}$ \\
\hline
\end{tabular}

(continua) 
Tabla 2 (continuación)

\begin{tabular}{|c|c|c|c|c|c|}
\hline Estudio & Diseño & $\begin{array}{l}\text { Tipo de } \\
\text { cáncer }\end{array}$ & Participantes (n) * & Intervención ** & Medición de resultados \\
\hline Saarto et al. 32 & $\begin{array}{l}\text { Ensayo clínico } \\
\text { aleatorizado }\end{array}$ & $\begin{array}{c}\text { Cáncer de } \\
\text { mama estadio } \\
\text { temprano (I-II) }\end{array}$ & $\begin{array}{l}\text { Características del tratamiento } \\
\text { de cáncer } \\
\text { Quimioterapia y } \\
\text { radioterapia }=500 \\
\text { Femenino }=500 \\
\text { Grupo experimental = 263 } \\
\text { Edad (años) }=52,3(36-68) \\
\text { Grupo control }=237 \\
\text { Edad (años) }=52,4(35-68)\end{array}$ & $\begin{array}{c}\text { Grupo experimental = } \\
\text { ejercicio aeróbico } \\
\text { Tiempo }=48 \text { semanas } \\
\text { Duración }=60 \text { minutos/sesión } \\
\text { Frecuencia }=1 \text { sesión/semana } \\
\text { Intensidad }=86 \%-92 \% \\
\text { Grupo control = estimular a } \\
\text { mantener su nivel previo de } \\
\text { actividad física y los hábitos de } \\
\text { ejercicio }\end{array}$ & $\begin{array}{l}\text { EORTC QLQ-C30, } \\
\text { FACIT-F, RBDI, WHQ }\end{array}$ \\
\hline Segal et al. 33 & $\begin{array}{l}\text { Ensayo clínico } \\
\text { aleatorizado }\end{array}$ & $\begin{array}{c}\text { Cáncer de } \\
\text { mama estadio } \\
\text { temprano (I-II) }\end{array}$ & $\begin{array}{l}\text { Características del tratamiento } \\
\text { de cáncer } \\
\text { Quimioterapia }=123 \\
\text { Femenino }=123 \\
\text { Grupo experimental }=42 \\
\text { Edad (años) }=51,4(8,7) \\
\text { Grupo control }=41 \\
\text { Edad (años) }=50,3(8,7) \\
\text { Grupo de ejercicio } \\
\text { auto-dirigido }=40 \\
\text { Edad (años) }=51,0(8,7)\end{array}$ & $\begin{array}{c}\text { Grupo experimental = } \\
\text { ejercicio aeróbico } \\
\text { Tiempo }=26 \text { semanas } \\
\text { Duración }=\text { no reportada } \\
\text { Frecuencia }=3 \text { sesiones } / \text { semana } \\
\text { Intensidad }=50 \%-60 \% \\
\text { Grupo control = cuidado } \\
\text { convencional } \\
\text { Grupo de ejercicio auto-dirigido = } \\
5 \text { veces por semana, progresivas, } \\
\text { caminando al } 50 \% \text { a } 60 \% \\
\text { del consumo de oxígeno } \\
\text { máximo pre dicho }\end{array}$ & $\begin{array}{l}\text { FACT-G, FACT-B, MOS } \\
\text { SF-36 }\end{array}$ \\
\hline Segal et al. 34 & $\begin{array}{l}\text { Ensayo clínico } \\
\text { aleatorizado }\end{array}$ & $\begin{array}{l}\text { Cáncer de } \\
\text { próstata, } \\
\text { etapas I-IV }\end{array}$ & $\begin{array}{c}\text { Características del tratamiento } \\
\text { de cáncer } \\
\text { Radioterapia }=121 \\
\text { Masculino }=121 \\
\text { Grupo experimental }=40 \\
\text { Edad (años) }=66,2(6,8) \\
\text { Resistencia }=40 \\
\text { Edad (años) }=66,4(7,6) \\
\text { Grupo control }=41 \\
\text { Edad (años) }=65,3(7,6)\end{array}$ & $\begin{array}{c}\text { Grupo experimental = ejercicio } \\
\text { aeróbico, entrenamiento de } \\
\text { resistencia y estiramiento } \\
\text { Tiempo = } 24 \text { semanas } \\
\text { Duración = } 45 \text { minutos/sesión } \\
\text { Frecuencia = } 3 \text { sesiones/semana } \\
\text { Intensidad = 70\%-75\% } \\
\text { Resistencia = entrenamiento } \\
\text { de resistencia supervisado de } 3 \\
\text { veces/semana por } 24 \text { semanas y } \\
2 \text { tiempos de } 8 \text { a } 12 \text { repeticiones } \\
\text { de } 10 \text { ejercicios al } 60 \% \text { a } 70 \% \\
\text { estimado de la } 1 \text { RM } \\
\text { Grupo control = cuidado } \\
\text { convencional }\end{array}$ & $\begin{array}{c}\text { FACT-G, FACT-P, FACT-F, } \\
\text { VO(2)max, 1RM, DEXA } \\
\text { scan (porcentaje de } \\
\text { grasa corporal) }\end{array}$ \\
\hline
\end{tabular}

1RM: test de resistencia muscular; BDI: Inventario de Depresión de Beck; DEXA: absorciometría de rayos X de doble energía; EORTC QLQ-C30: Organización Europea para la Investigación y Tratamiento del Cáncer de Calidad de Vida; FACIT-F: Evaluación Funcional del Tratamiento de Enfermedades Crónicas, cuestionario para la fatiga; FACT-B: Evaluación Funcional del Tratamiento del Cáncer, HECHO - mama; FACT-F: Evaluación Funcional del Tratamiento del Cáncer, fatiga; FACT-G: Evaluación Funcional del Tratamiento del Cáncer, el general; FACT-P: Escala de Fatiga de la Evaluación Funcional de la Terapia del Cáncer - módulo de Próstata; MOS SF-36: Cuestionario Breve de Resultados Médicos; PANAS: Cuestionario Escocés de Actividad Física; PARQ: Cuestionario de Preparación de Actividad Física; PFS: Escala de Fatiga de Piper; RBDI: Versión finlandesa modificada de la Escala de Depresión de 13 Ítems de Beck; SCFS: Escala de Schwartz de la Fatiga por Cáncer; SPAQ: Inventario de Afecto Positivo y Negativo; SWLS: Escala de Satisfacción con la Vida; WHQ: Cuestionario de Salud de la Mujer; VO(2)max: consumo máximo de oxígeno.

* Edad se presenta como media y desviación estándar.

** Intervenciones de actividad física supervisadas, normalmente, consistían en un período de calentamiento, entrenamiento aeróbico (caminar, circuitos en bicicleta), entrenamiento de la fuerza muscular (pecho y las piernas), ejercicios de estiramiento, periodos de enfriamiento y relajación. 


\section{Características de las intervenciones con ejercicio físico}

Las intervenciones con ejercicio físico tuvieron una duración media de 16,5 $\pm 12,3$ semanas con un promedio de tres sesiones de entrenamiento por semana y una duración promedio de $45,0 \pm 29,1$ minutos por sesión. La intensidad del entrenamiento varió considerablemente entre los estudios, oscilando entre un 50\%-70\% de la frecuencia cardíaca máxima. En lo que respecta a la modalidad de ejercicio físico prescrita, cinco estudios evaluaron los efectos del entrenamiento de resistencia $24,29,30,31,34$, mientras que los programas de estiramiento muscular fueron evaluados en cuatro estudios 24,29,30,34 (Tabla 2).

\section{Reporte de eventos adversos}

Tres estudios reportaron eventos adversos, relacionados a las intervenciones con ejercicio físico, en pacientes con cáncer sometidos a tratamiento activo 26,27,33. En el estudio de Courneya et al. 26 se presentaron cinco eventos adversos en el grupo intervenido con ejercicio físico (linfedema, problemas ginecológicos y gripe). Segal et al. 33 reportaron tres eventos adversos, de los cuales uno resultó en hospitalización, un caso de dolor torácico y en el grupo control se dio un episodio de síncope sin complicaciones mayores. Finalmente, Courneya et al. 27 observaron tres casos de dolor lumbar, de rodilla y de cadera en el grupo de ejercicio físico.

Estimaciones de efecto de las intervenciones con ejercicio físico en la fatiga de pacientes con cáncer sometidos a tratamiento activo

Los 11 estudios incluidos brindaron estimaciones de efecto consistentes para los procedimientos de meta-análisis 24,25,26,27,28,29,30,31,32,33,34. En el modelo de efectos aleatorios se encontró que el ejercicio físico redujo de manera significativa los niveles de fatiga de pacientes con cáncer sometidos a tratamiento activo (SMD = -3,0; IC95\%: $-5,21 ;-0,80), p<0,0001$. Se detectó un alto nivel de heterogeneidad estadística ( $\mathrm{I}^{2}=81,3 \%$; $<0,0001$ ) (Figura 2). El sesgo de publicación se evaluó con los datos obtenidos en este desenlace para el cual se obtuvo moderada evidencia de reportes selectivos de los resultados, con base en la observación de la estimación de efecto ponderada (Figura 3$)$.

Figura 2

Efectividad del ejercicio físico en el control de la fatiga relacionada al cáncer durante el tratamiento activo.

\begin{tabular}{|c|c|c|}
\hline Estudio & SMD (IC95\%) & Peso (\%) \\
\hline Adamsen et al. ${ }^{24}$ & $-6,40(-12,41 ;-0,39)$ & 7,21 \\
\hline Campbell et al. ${ }^{25}$ & $-1,86(-4,03 ; 0,31)$ & 13,51 \\
\hline Courneya et al. ${ }^{26}$ & $6,80(1,16 ; 12,44)$ & 7,71 \\
\hline Courneya et al. ${ }^{27}$ & $-2,50(-6,15 ; 1,15)$ & 10,91 \\
\hline Dimeo et al. ${ }^{28}$ & $-5,00(-16,14 ; 6,14)$ & 3,13 \\
\hline Galvão et al. ${ }^{29}$ & $-15,80(-24,03 ;-7,57)$ & 4,91 \\
\hline Milne et al. ${ }^{30}$ & $-5,50(-7,57 ;-3,43)$ & 13,67 \\
\hline Mutrie et al. ${ }^{31}$ & $-4,30(-7,64 ;-0,96)$ & 11,45 \\
\hline Saarto et al. ${ }^{32}$ & $-0,40(-0,82 ; 0,02)$ & 15,45 \\
\hline Segal et al. ${ }^{33}$ & $-3,80(-15,54 ; 7,94)$ & 2,88 \\
\hline Segal et al. ${ }^{34}$ & $-2,10(-6,77 ; 2,57)$ & 9,16 \\
\hline Total $\left(\left.\right|^{2}=81,3 \% ; p=0,000\right)$ & $-3,00(-5,21 ;-0,80)$ & 100,00 \\
\hline
\end{tabular}

IC95\%: intervalo de confianza del 95\%; SMD: diferencias de medias estandarizadas.

Nota: los pesos están relacionados con el análisis de efectos aleatorios. 


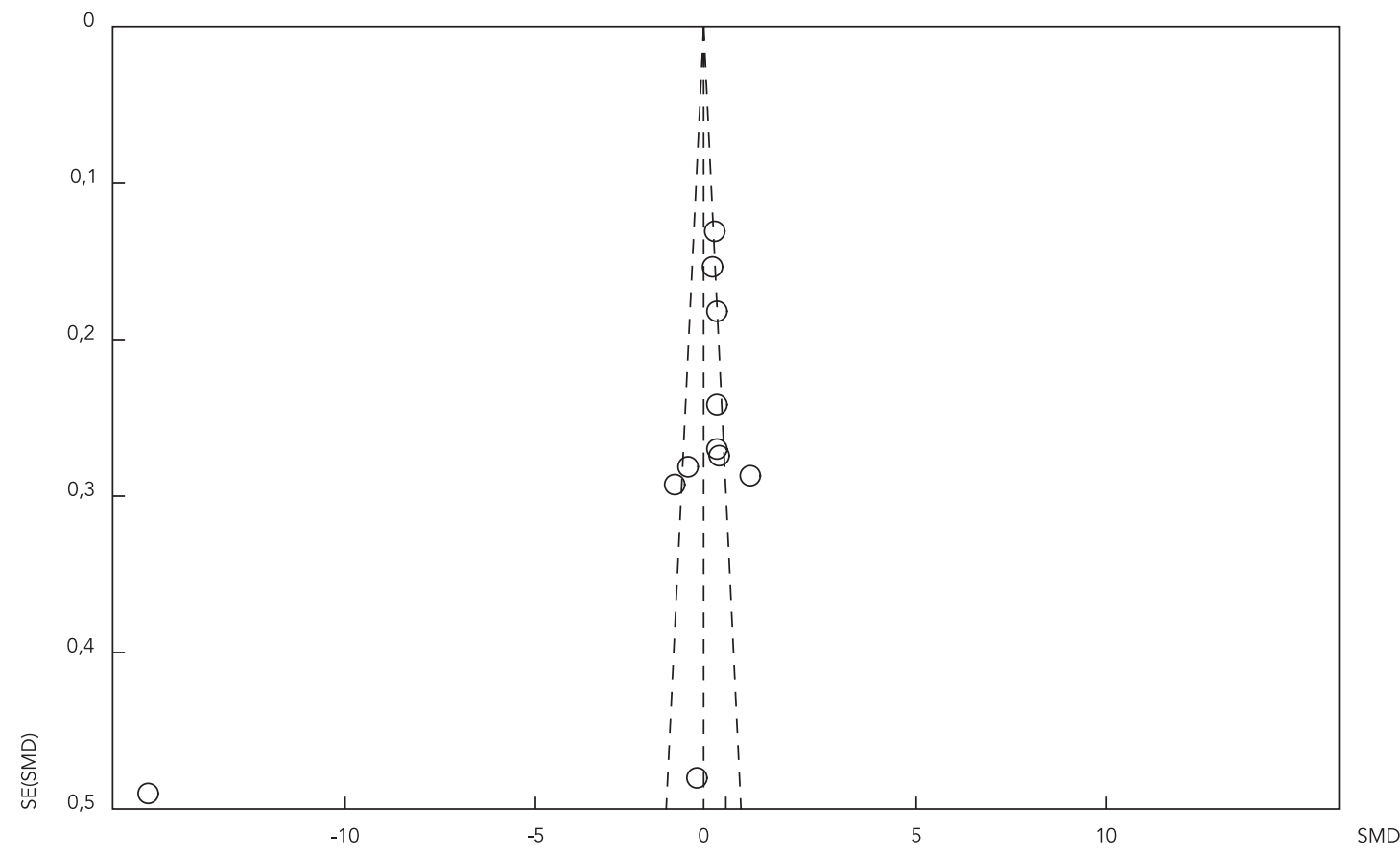

SE: error estander; SMD: diferencias de medias estandarizadas.

Nota: los pesos están relacionados con el análisis de efectos aleatorios.

\section{Análisis de subgrupos según modalidad de ejercicio físico}

Seis estudios evaluaron el efecto del entrenamiento de resistencia en la fatiga de pacientes con cáncer durante el tratamiento activo $24,25,29,30,31,34$. Dos estudios evaluaron el efecto del ejercicio de resistencia combinado con entrenamiento aeróbico 25,31, mientras que los cuatro estudios restantes incluyeron protocolos de entrenamiento de resistencia, aeróbico y períodos de estiramiento al final de la sesión de ejercicio físico 24,29,30,34. El análisis combinado, calculado mediante el modelo de efectos aleatorios, mostró diferencias estadísticamente significativas a favor del entrenamiento de resistencia (fortalecimiento muscular) en comparación con la no intervención (SMD = -4,5; IC95\%: -7,24; -1,82), $\mathrm{p}=0,001$. El nivel de heterogeneidad estadística fue moderado ( $\left.\mathrm{I}^{2}=69,6 \% ; \mathrm{p}=0,006\right)$ (Figura 4 ).

\section{Análisis de subgrupos, según localización} del cáncer

El análisis de los efectos del ejercicio físico en la fatiga de pacientes con cáncer de mama, sometidos a tratamiento activo 25,26,30,31,32,33, mostró diferencias estadísticamente significativas en favor del ejercicio físico, comparado con la no intervención (SMD = -0,49; IC95\%: -0,90; -0,08), $\mathrm{p}=0,01$, con moderada heterogeneidad estadística ( $\left.\mathrm{I}^{2}=64 \% ; \mathrm{p}=0,01\right)$. De igual forma, se observaron cambios significativos en los pacientes con cáncer de próstata (SMD = -5,4; IC95\%: -9,4; -1,3), $\mathrm{p}=0,009$, con alta heterogeneidad estadística (I2 $=87,6 \% ; \mathrm{p}=0,005)$.

\section{Efectos del ejercicio físico en las medidas de resultado secundarias}

El bienestar físico fue investigado en siete estudios 24,26,28,29,30,31,33, para los cuales el análisis combinado, según un modelo de efectos aleatorios, no mostró diferencias estadísticamente significativas a favor del ejercicio físico en 


\begin{tabular}{|c|c|c|c|}
\hline Estudio & & SMD (IC95\%) & Peso (\%) \\
\hline Adamsen et al. ${ }^{24}$ & & $-6,40(-12,41 ;-0,39)$ & 11,69 \\
\hline Campbell et al. ${ }^{25}$ & $\rightarrow-$ & $-1,49(-3,66 ; 0,68)$ & 23,42 \\
\hline Galvão et al. ${ }^{29}$ & & $-15,80(-24,03 ;-7,57)$ & 7,78 \\
\hline Milne et al. ${ }^{30}$ & $\rightarrow$ & $-5,50(-7,57 ;-3,43)$ & 23,74 \\
\hline Mutrie et al. ${ }^{31}$ & & $-3,20(-6,90 ; 0,50)$ & 18,21 \\
\hline Segal et al. ${ }^{34}$ & & $-2,10(-6,77 ; 2,57)$ & 15,16 \\
\hline Total $\left(1^{2}=69,6 \% ; p=0,006\right)$ & & $-4 ., 53(-7,24 ;-1,82)$ & 100,00 \\
\hline 工 & & & \\
\hline
\end{tabular}

IC95\%: intervalo de confianza del 95\%; SMD: diferencias de medias estandarizadas.

Nota: los pesos están relacionados con el análisis de efectos aleatorios.

comparación con la no intervención $(\mathrm{SMD}=$ 0,27; IC95\%: -0,19; 0,74), p >0,05, en presencia de un alto nivel de heterogeneidad estadística ( ${ }^{2}=$ $88 \%), p=0,001$. De manera similar, los efectos del ejercicio físico sobre el bienestar funcional de pacientes con cáncer durante el tratamiento activo fueron reportados por seis estudios 24,26,28,29,30,31, cuyo análisis combinado no arrojó diferencias significativas (SMD = 0,47; IC95\%: $-0,00 ; 0,95$ ), $\mathrm{p}>0,05$; se observó una alta heterogeneidad estadística $\left(\mathrm{I}^{2}=87 \%\right)$. Esta carencia de significancia estadística puede ser atribuida a la heterogeneidad en las intervenciones y a la medición de estos desenlaces.

\section{Discusión}

Los hallazgos del presente metaanálisis muestran que el ejercicio físico supervisado mejora los niveles de fatiga en pacientes con cáncer durante el tratamiento activo. Con relación al seguimiento, Courneya et al. 26 encontraron que las diferencias en la fatiga, el funcionamiento físico y la calidad de vida no fueron significativas a los 6 meses de medición post-intervención $(p=0,054)$. Estos resultados contrastan con lo descrito por Ferrer et al. 36 , quienes plantean que las intervenciones con ejercicio físico y su consiguiente seguimiento mejoran la calidad de vida, fatiga y funcionalidad física de los sobrevivientes de cáncer (espe- cialmente en las mujeres) con independencia del tiempo de seguimiento. Por otra parte, Milne et al. 30 obtuvieron igualmente mejoras estadísticamente significativas en la fatiga, calidad de vida, ansiedad y el fitness físico ( $\mathrm{p}<0,05)$, y Mutrie et al. 31 encontraron que los efectos del ejercicio físico en la fatiga del grupo experimental se mantuvieron durante los seis meses de seguimiento. Estos resultados coinciden con lo reportado en los estudios de Fong et al. 37 y Zeng et al. 38, quienes concluyen que la actividad física reduce el cansancio y los síntomas depresivos e incrementa la calidad de vida en mujeres sobrevivientes de cáncer de mama; destacando que el análisis de subgrupo del presente metaanálisis mostró mejoras significativas en la fatiga de pacientes con cáncer de mama.

Algunos eventos adversos derivados de las intervenciones con ejercicio físico en pacientes con cáncer sometidos a tratamiento activo fueron reportados en los estudios incluidos. Estos eventos incluyeron desde linfedema, problemas ginecológicos, gripe dolor lumbar, de rodilla y de cadera como describe Courneya et al. 26,27; o dolor torácico y síncope según Segal et al. 33. En cualquier caso, los diferentes estudios analizados plantean que estos eventos adversos, más que una consecuencia del ejercicio físico, son el resultado de la baja forma física que muchos de estos pacientes poseen. 
Con relación al efecto de las intervenciones con ejercicio físico sobre la fatiga en pacientes con cáncer sometidos a tratamiento activo, en los estudios analizados $24,25,26,27,28,29,30,31,32,33,34$ el ejercicio físico redujo de manera significativa los niveles de fatiga en estos pacientes. Estos resultados coinciden con lo descrito por otros estudios, como el publicado por Tomlinson et al. 39, quienes a partir de un metaanálisis de ensayos controlados aleatorios, obtienen que el ejercicio físico posee un efecto moderado en la reducción de la fatiga, en comparación con una intervención de control, concluyendo que el ejercicio es eficaz para el tratamiento de la fatiga relacionada con el cáncer. En esta misma línea, Puetz \& Herring 40 concluyen que el ejercicio físico reduce la fatiga relacionada con el cáncer en los pacientes durante y después del tratamiento quimioterapéutico, presentando un efecto paliativo en los pacientes durante el tratamiento y un efecto de recuperación post-tratamiento.

Por otra parte, atendiendo a la modalidad de ejercicio físico, seis de los estudios incluidos en este meta-análisis 24,25,29,30,31,34 ponen de manifiesto el efecto positivo que el entrenamiento de resistencia ejerce sobre la fatiga en pacientes oncológicos durante la fase de tratamiento activo. Estos resultados coinciden con lo descrito por Velthuis et al. ${ }^{41}$, quienes observaron que una intervención con entrenamiento de resistencia durante 18 semanas reducía de manera significativa los niveles de fatiga en pacientes con cáncer de mama y cáncer de colon en fase de tratamiento activo. De manera similar, Winters-Stone et al. 42 concluyen que el entrenamiento de resistencia no sólo reduce la fatiga en pacientes oncológicos, sino que además disminuye la posibilidad de sufrir caídas accidentales en estos pacientes. Si bien, otros estudios como el desarrollado por Galanti et al. ${ }^{43}$ plantean que tan importante es la modalidad de ejercicio como la intensidad del mismo, proponiendo la realización de ejercicio físico aeróbico a dos niveles de intensidad para reducir la fatiga, esto es, a baja intensidad $(40 \%$ de la frecuencia cardíaca máxima) y moderada intensidad (60\% de la frecuencia cardíaca máxima). Pese a lo anterior, es importante destacar que no fue posible calcular el efecto específico del entrenamiento de resistencia en la fatiga de los pacientes con cáncer sometidos a tratamiento activo, ya que en los estudios incluidos se prescribieron protocolos combinados de ejercicio aeróbico y de resistencia, algunos con períodos de estiramiento muscular. Se requiere de estudios adicionales que permitan establecer consenso en este campo de estudio.

Los efectos del ejercicio físico sobre la fatiga en pacientes con cáncer de mama bajo tra- tamiento activo fueron estadísticamente significativos en el análisis ponderado, ya que los estudios analizados 25,26,30,31,32,33 muestran diferencias significativas en favor del ejercicio físico, comparado con la no intervención. Estos resultados coinciden con lo descrito por otros autores en pacientes con cáncer de mama, así como con otras localizaciones, tales como próstata 35 y colon 44 . Sin embargo, se requiere de estudios adicionales que favorezcan el consenso en la literatura científica acerca de los efectos y seguridad del ejercicio físico en estas pacientes.

Con relación a los efectos del ejercicio físico sobre el bienestar físico de estos pacientes, el análisis de los estudios incluidos en este metaanálisis 24,26,28,29,30,31,33 no mostró diferencias estadísticamente significativas a favor del ejercicio físico en comparación con la no intervención. Estos resultados no concuerdan con lo descrito por Mehnert et al. 45, quienes demuestran que una intervención de diez semanas de ejercicio físico aeróbico mejora significativamente el bienestar físico y la fatiga en mujeres con cáncer de mama después de un período de cuatro semanas de tratamiento con quimioterapia. En el caso de los efectos del ejercicio físico sobre el bienestar funcional en pacientes con cáncer durante la fase de tratamiento activo, el análisis de los estudios analizados 24,26,28,29,30,31 no arrojó diferencias significativas. Estos resultados son contrarios a lo descrito por Mishra et al. 46, quienes afirman que el ejercicio físico posee efectos beneficiosos sobre el bienestar funcional y social así como sobre la fatiga. En este estudio, los autores concluyen además que tales efectos son más pronunciados cuando el ejercicio físico es de moderada a vigorosa intensidad.

Aunque los efectos del ejercicio de resistencia no han sido descritos claramente por la ASCO 47, existen pruebas de los posibles efectos en la percepción de fatiga en personas con cáncer de mama 48,49 . Sin embargo, para entender los posibles mecanismos del beneficio de este tipo de entrenamiento, se requiere de información adicional sobre los efectos de la quimioterapia y la radiación en las células satélite musculares o progenitoras que se activan en respuesta al entrenamiento de fuerza. Tampoco está claro si las personas previamente sedentarias pueden adherirse a un programa de ejercicios de resistencia en otros síntomas relacionados a la fatiga como ansiedad, depresión, falta de sueño o estado de ánimo.

Como ya se ha mencionado, la diversidad de nuestros resultados pueden ser explicados por características propias del diseño metodológico, donde la combinación estadística de datos provenientes de diferentes estudios, con una alta 
heterogeneidad, conlleva a variaciones en las estimaciones de efecto.

\section{Limitaciones}

El presente estudio presenta algunas limitaciones que deben ser reportadas. Las estimaciones de efecto presentaron altas cifras de heterogeneidad estadística, lo cual sugiere una interpretación cauta de los hallazgos encontrados. Es importante destacar que esta heterogeneidad fue abordada mediante un modelo de efectos aleatorios del inverso de la varianza. También se evidenciaron variaciones importantes en los protocolos de ejercicio en los estudios incluidos, lo cual refleja una heterogeneidad metodológica a considerar y limita la recomendación de un programa de entrenamiento específico. Se sugiere adoptar las recomendaciones actuales para la prescripción de ejercicio físico en pacientes con cáncer 50 . No se publicó un protocolo previo de la presente revisión sistemática; dado que los investigadores no realizaron cambios considerables a la metodología propuesta inicialmente, y no se compromete la consistencia metodológica del estudio.
Con base en todo lo anterior, se pone de manifiesto la necesidad de futuras investigaciones para esclarecer aún más los efectos positivos del ejercicio, así como su permanencia en el tiempo frente a síntomas clínicos tan prevalentes como es la fatiga en el paciente oncológico. Las próximas investigaciones experimentales han de incluir metodologías más uniformes, tanto en sus intervenciones, como en la medición de sus resultados.

\section{Conclusión}

El ejercicio físico es una intervención segura y eficaz en el manejo de la fatiga relacionada con el cáncer en pacientes sometidos a tratamiento activo. Estos hallazgos destacan la importancia de la prescripción del ejercicio físico en la rehabilitación oncológica, en especial del entrenamiento de la fuerza muscular; para así potenciar los efectos terapéuticos del tratamiento anti-cáncer. Se requiere de futuras investigaciones que consoliden el consenso acerca de los efectos y seguridad de las intervenciones con ejercicio físico en pacientes con cáncer y que promuevan su recomendación por parte de los profesionales de la salud.

\section{Resumo}

O objetivo foi determinar a efetividade do exercício físico sobre a fadiga em pacientes com câncer durante o tratamento ativo. As bases de dados PubMed Central, EMBASE e OVID foram consultadas até abril de 2014 para identificar ensaios clínicos randomizados que avaliaram o efeito do exercício sobre a fadiga em pacientes com câncer em tratamento ativo. Onze estudos ( $n=1.407)$ foram incluídos. A quimioterapia foi o tratamento mais comum $(n=1.028)$. Os estudos tiveram baixo risco de viés e alta qualidade metodológica. As es- timativas de efeito mostraram que o exercício melhorou significativamente a fadiga (DMP = -3,0; IC95\%: $-5,21$; $-0,80), p<0,0001$. Efeitos semelhantes sobre o treinamento de resistência (DMP $=-4,5$; IC95\%: -7,24; - 1,82), $p=0,001$ foram encontrados. O exercício físico é uma intervenção segura e eficaz contra a fadiga em pacientes submetidos ao tratamento ativo.

Exercício; Fadiga; Neoplasias; Quimioterapia 


\section{Colaboradores}

J. F. Meneses-Echávez participó en la concepción del estudio, construcción y aplicación de las estrategias de búsqueda, criterios de selección, evaluación de la calidad metodológica y el riesgo de sesgo, análisis estadístico y elaboración del artículo científico. E. GonzálezJiménez colaboró en la evaluación de los criterios de selección, evaluación de la calidad metodológica y el riesgo de sesgo, extracción de datos y elaboración del artículo científico. R. Ramírez-Vélez y J. Schmidt-RíoValle colaboraron en la aplicación de las estrategias de búsqueda, criterios de selección y elaboración del artículo científico. J. E. Correa-Bautista participó en la aplicación de la estrategia de búsqueda, evaluación de la calidad metodológica y el riesgo de sesgo, extracción de datos y elaboración del artículo científico.

\section{Referencias}

1. Siegel R, Naishadham D, Jemal A. Cancer statistics. Cancer J Clin 2013; 63:11-30.

2. Murillo R, Piñeros M, Hernández G. Atlas de mortalidad por cáncer en Colombia. Bogotá: Instituto Nacional de Cancerología/Instituto Geográfico Agustín Codazzi; 2004.

3. Murillo R, Quintero A, Piñeros M, Bravo MM, Cendales R, Wiesner C, et al. Modelo para el control del cáncer en Colombia. Bogotá: Escala; 2006. (Serie de Documentos Técnicos INC, 1).

4. Hardee JP, Porter RR, Sui X, Archer E, Lee IM, Lavie CJ, et al. The effect of resistance exercise on all-cause mortality in cancer survivors. Mayo Clin Proc 2014; 89:1108-15.

5. Newton RU, Galvão DA. Exercise in prevention and management of cancer. Curr Treat Options Oncol 2008; 9:135-46.

6. Stone P, Richardson A, Ream E, Smith A, Kerr D, Kearney N. Cancer related fatigue, inevitable, unimportant and untreatable? Results of a multi-centre patient survey. Ann Oncol 2000; 11:971-5.

7. National Comprehensive Cancer Network. Clinical practice guidelines in oncology. http://www.nccn. org/professionals/physician_gls/pdf/fatigue.pdf (accedido el 19/Abr/2014).

\section{Agradecimientos}

El presente trabajo forma parte del Proyecto Práctica del autoexamen de seno y los conocimientos, factores de riesgo y estilos de vida relacionados con el cáncer de mama en mujeres jóvenes de la Universidad Santo Tomás de Bogotá: un análisis transversal (9a Convocatoria FODEIN- Código del proyecto 4110060001 - 008).
8. Hartvig P, Aulin J, Hugerth M, Wallenberg S, Wagenius G. Fatigue in cancer patients treated with cytotoxic drugs. J Oncol Pharm Pract 2006; 12: 155-64.

9. Groenvold M, Petersen MA, Idler E, Bjorner JB, Fayers PM, Mouridsen HT. Psychological distress and fatigue predicted recurrence and survival in primary breast cancer patients. Breast Cancer Res Treat 2007; 105:209-19.

10. Brown JC, Huedo-Medina TB, Pescatello LS, Pescatello SM, Ferrer RA, Johnson BT. Efficacy of exercise interventions in modulating cancer-related fatigue among adult cancer survivors: a metaanalysis. Cancer Epidemiol Biomarkers Prev 2011; 20:123-33.

11. Strasser B, Steindorf K, Wiskemann J, Ulrich CM. Impact of resistance training in cancer survivors: a meta-analysis. Med Sci Sports Exerc 2013; 45: 2080-90.

12. Visovsky C. Muscle strength, body composition, and physical activity in women receiving chemotherapy for breast cancer. Integr Cancer Ther 2006; 5:183-91.

13. Cramp F, Byron J. Exercise for the management of cancer-related fatigue in adults. Cochrane Database Syst Rev 2012; 11:CD006145. 
14. Lin KY, Shun SC, Lai YH, Liang JT, Tsauo JY. Comparison of the effects of a supervised exercise program and usual care in patients with colorectal cancer undergoing chemotherapy. Cancer Nurs 2014; 37:E21-9.

15. Schneider CM, Hsieh CC, Sprod LK, Carter SD, Hayward R. Effects of supervised exercise training on cardiopulmonary function and fatigue in breast cancer survivors during and after treatment. Cancer 2007; 110:918-25.

16. Velthuis MJ, Agasi-Idenburg SC, Aufdemkampe G, Wittink HM. The effect of physical exercise on cancer-related fatigue during cancer treatment: a meta-analysis of randomised controlled trials. Clin Oncol 2010; 22:208-21.

17. Liberati A, Altman DG, Tetzlaff J, Mulrow C, Gøtzsche PC, Ioannidis JP, et al. The PRISMA statement for reporting systematic reviews and metaanalyses of studies that evaluate health care interventions: explanation and elaboration. J Clin Epidemiol 2009; 62:1-34.

18. Higgins JPT, Green S. Cochrane handbook for systematic reviews of interventions. Version 5.1.0 http://handbook.cochrane.org/ (accedido el 15/ Dic/2013).

19. Wolin KY, Schwartz AL, Matthews CE, Courneya KS, Schmitz KH. Implementing the exercise guidelines for cancer survivors. J Support Oncol 2012; 10:171-7.

20. Schmitz KH, Courneya KS, Matthews C, DemarkWahnefried W, Galvão DA, Pinto BM, et al. American College of Sports Medicine roundtable on exercise guidelines for cancer survivors. Med Sci Sports Exerc 2010; 42:1409-26.

21. De Morton NA. The PEDro scale is a valid measure of the methodological quality of clinical trials: a demographic study. Aust J Physiother 2009; 55:129-33.

22. Moseley AM, Herbert RD, Sherrington C, Maher CG. Evidence for physiotherapy practice: a survey of the Physiotherapy Evidence Database (PEDro). Aust J Physiother 2002; 48:43-9.

23. Higgins JP, Thompson SG, Deeks JJ, Altman DG. Measuring inconsistency in meta-analyses. BM] 2003; 327:557-60.

24. Adamsen L, Quist M, Andersen C, Møller T, Herrstedt J, Kronborg D, et al. Effect of a multimodal high intensity exercise intervention in cancer patients undergoing chemotherapy, randomised controlled trial. BMJ 2009; 339:b3410.

25. Campbell A, Mutrie N, White F, McGuire F, Kearney N. A pilot study of a supervised group exercise programme as a rehabilitation treatment for women with breast cancer receiving adjuvant treatment. Eur J Oncol Nurs 2005; 9:56-63.

26. Courneya KS, Mackey JR, Bell GJ, Jones LW, Field CJ, Fairey AS. Randomized controlled trial of exercise training in postmenopausal breast cancer survivors, cardiopulmonary and quality of life outcomes. J Clin Oncol 2003; 21:1660-8.

27. Courneya KS, Sella CM, Stevinson C, McNeely ML, Peddle CJ, Friedenreich CM, et al. Randomized controlled trial of the effects of aerobic exercise on physical functioning and quality of life in lymphoma patients. J Clin Oncol 2009; 27:4605-12.
28. Dimeo FC, Thomas F, Raabe-Menssen C, Mathias $M$. Effect of aerobic exercise and relaxation training on fatigue and physical performance of cancer patients after surgery. A randomised controlled trial. Support Care Cancer 2004; 12:774-9.

29. Galvão DA, Taaffe DR, Spry N, Joseph D, Newton RU. Combined resistance and aerobic exercise program reverses muscle loss in men undergoing androgen suppression therapy for prostate cancer without bone metastases, a randomized controlled trial. J Clin Oncol 2010; 28:340-7.

30. Milne HM, Wallman KE, Gordon S, Courneya KS Effects of a combined aerobic and resistance exercise program in breast cancer survivors, a randomized controlled trial. Breast Cancer Res Treat 2008; 108:279-88.

31. Mutrie N, Campbell AM, Whyte F, McConnachie A, Emslie C, Lee L, et al. Benefits of supervised group exercise programme for women being treated for early stage breast cancer, pragmatic randomised controlled trial. BMJ 2007; 334:517.

32. Saarto T, Penttinen HM, Sievänen H, KellokumpuLehtinen PL, Hakamies-Blomqvist L, Nikander R, et al. Effectiveness of a 12-month exercise program on physical performance and quality of life of breast cancer survivors. Anticancer Res 2012; 32:3875-84.

33. Segal RJ, Evans W, Johnson D, Smith J, Colletta S, Gayton J, et al. Structured exercise improves physical functioning in women with stages I and II breast cancer: results of a randomized controlled trial. J Clin Oncol 2001; 19:657-65.

34. Segal RJ, Reid RD, Courneya KS, Sigal RJ, Kenny GP, Prud'Homme DG, et al. Randomized controlled trial of resistance or aerobic exercise in men receiving radiation therapy for prostate cancer. J Clin Oncol 2009; 27:344-51.

35. Courneya KS, Segal RJ, Reid RD, Jones LW, Malone SC, Venner PM, et al. Three independent factors predicted adherence in a randomized controlled trial of resistance exercise training among prostate cancer survivors. J Clin Epidemiol 2004; 57: 571-9.

36. Ferrer RA, Huedo-Medina TB, Johnson BT, Ryan S, Pescatello LS. Exercise interventions for cancer survivors: A meta-analysis of quality of life outcomes. Ann Behav Med 2011; 41:32-47.

37. Fong DY, Ho JW, Hui BP, Lee AM, Macfarlane DJ, Leung SS, et al. Physical activity for cancer survivors: meta-analysis of randomised controlled trials. BMJ 2012; 344:e70.

38. Zeng Y, Huang M, Cheng AS, Zhou Y, So WK. Metaanalysis of the effects of exercise intervention on quality of life in breast cancer survivors. Breast Cancer 2014; 21: 262-74.

39. Tomlinson D, Diorio C, Beyene J, Sung L. Effect of exercise on cancer-related fatigue: a meta-analysis. Am J Phys Med Rehabil 2014; 93:675-86.

40. Puetz TW, Herring MP. Differential effects of exercise on cancer-related fatigue during and following treatment: a meta-analysis. Am J Prev Med 2012; 43:e1-24. 
41. Velthuis MJ, May AM, Koppejan-Rensenbrink RA, Gijsen BC, van Breda E, de Wit GA, et al. Physical Activity during Cancer Treatment (PACT) Study: design of a randomised clinical trial. BMC Cancer 2010; 10:272.

42. Winters-Stone KM, Dobek J, Bennett JA, Nail LM, Leo MC, Schwartz A. The effect of resistance training on muscle strength and physical function in older, postmenopausal breast cancer survivors: a randomized controlled trial. J Cancer Surviv 2012; 6:189-99.

43. Galanti G, Stefani L, Gensini G. Exercise as a prescription therapy for breast and colon cancer survivors. Int J Gen Med 2013; 6:245-51.

44. Lee DH, Kim JY, Lee MK, Lee C, Min JH, Jeong DH, et al. Effects of a 12-week home-based exercise program on the level of physical activity, insulin, and cytokines in colorectal cancer survivors: a pilot study. Support Care Cancer 2013; 21:2537-45.

45. Mehnert A, Veers S, Howaldt D, Braumann KM, Koch U, Schulz KH. Effects of a physical exercise rehabilitation group program on anxiety, depression, body image, and health-related quality of life among breast cancer patients. Onkologie 2011; 34:248-53.
46. Mishra SI, Scherer RW, Snyder C, Geigle PM, Berlanstein DR, Topaloglu O. Exercise interventions on health-related quality of life for people with cancer during active treatment. Cochrane Database Syst Rev 2012; 8:CD008465.

47. American Cancer Society. Cancer facts \& figures. Atlanta: American Cancer Society; 2012.

48. Meneses-Echávez JF, González-Jiménez E, Ramírez-Vélez R. Supervised exercise reduces cancer-related fatigue: a systematic review. J Physiother 2015; 61:3-9.

49. Meneses-Echávez JF, González-Jiménez E, Ramírez-Vélez R. Effects of supervised multimodal exercise interventions on cancer-related fatigue: systematic review and meta-analysis of randomized controlled trials. Biomed Res Int; in press.

50. Buffart LM, Galvão DA, Brug J, Chinapaw MJ, Newton RU. Evidence-based physical activity guidelines for cancer survivors: current guidelines, knowledge gaps and future research directions. Cancer Treat Rev 2014; 40:327-40.

Recibido el 30/Jul/2014

Versión final presentada el 07/Ene/2015

Aprobado el 14/Ene/2015 